\title{
Structural Feature Selection For English-Korean Statistical Machine Translation
}

\author{
Seonho Kim, Juntae Yoon, Mansuk Song \\ \{pobi, jtyoon, mssong\}@december.yonsci.ac.kr \\ Dept. of Computer Science, \\ Yonsci University, Scoul, Korca
}

\begin{abstract}
When aligning texts in very different languages such as Korcan and English, structural features beyond word or plirase give useful information. In this paper, we present a method for selecting structural features of two languages, from which we construct, a model that assigns the conditional probabilities to corresponding tag sequences in bilingual EnglishKorean corpora. For tag sequence mapping between two langauges, we first define a structural foature function which represents statistical properties of empirical distribution of a set of training samples. The system, based on maximum entropy concept, selects only features that produce high increases in loglikelihood of training samples. These structurally mapped features are more informative knowledge for statistical machinc translation between English and Korean. Also, the information can help to reduce the parameter space of statistical alignment by elinninating syntactically unlikely alignnents.
\end{abstract}

\section{Introduction}

Aligned texts have been used for derivation of bilingual dictionaries and terminology databases which are useful for machine translation and cross languages information retricval. Thus, a lot of alignment techniques have beon suggested at the sentence (Gale et al., 1993), phrase (Shin ot al., 1996), noun phrase (Kupiec, 1993), word (Brown et al., 1993; Berger ct al., 1996; Melamed, 1997), collocation (Smadja et, al., 1996) and torminology level.

Some work has used lexical association measures for word alignments. However, the association measures could be misled since a word in a source language frequently co-occurs with more than one word in a target language. In other work, iterative reestimation techniques have been employed. They were usually incorporated with the EM algorithm and dynamic programming. In that case, the probabilities of alignments usually served as parameters in a model of statistical machine translation.

In statistical machine translation, IBM $1 \sim 5$ models (Brown et al., 1993) based on the source-channel model have been widely used and revisod for many language domains and applications. It has also shortcoming that it necls much itcration time for parameter estimation and high decoding complexity, however.

Much work has been done to overcome the problem. Wu (1996) adopted channels that eliminate syntactically unlikely alignments and Wang ot al. (1998) presented a model based on structures of two languages. Tillmam of al. (1997) suggested the dynanic programming based scarch to select the best alignment and preprocessed bilingual texts to remove word order differences. Sato et al. (1998) and Och ot al. (1998) proposed a model for learning translation rules with morphological information and word catcgory in order to improve statistical translation.

Turthermore, many rescarches assumed onc-toone correspondence due to the complexity and computation time of statistical alignments. Although this assumption tumed ont to be useful for alignment of close languages such as English and French, it is not applicable to very different languages, in particular, Korean and English where there is rarely close correspondence in order at the word level. For such languages, even phrase level alignment, not to montion word alignment, does not gives good translation due to structural difference. Hence, structural features beyond word or phrase should be considered to get better translation between Bnglish and Korean. In addition, the construction of structural bilingual texts would be more informative for extracting linguistic knowledge.

In this paper, we suggest a method for structural mapping of bilingual language on the basis of the maximum entorpy and feature induction framework. Our model based on POS tag sequence mapping has two advantages: First, it can reduce a lot of parameters in statistical machine translation by eliminating syntactically unlikely alignments. Sccond, it can be used as a preprocessor for lexical alignments of bilingual corpora although it can be also exploited by itself for alignment. In this case, it would serve as the first step of alignment for reducing the parameter space. 


\section{Motivation}

In order to devise parameters for statistical modeling of translation, we started our research from the IBM model which has been widely used by many researches. The IBM model is represented with the formula shown in (1)

$$
p(\mathbf{f}, \mathbf{a} \mid \mathbf{e})=\prod_{i=1}^{l} \mathbf{n}\left(\phi_{i} \mid e_{i}\right) \prod_{j=1}^{m} \mathbf{t}\left(f_{j} \mid e_{a_{j}}\right) \mathbf{d}\left(j \mid a_{j}, m, l\right)
$$

Here, $\mathbf{n}$ is the fertility probability that an English word generates $n$ French words, $\mathrm{t}$ is the alignment probability that the English word $e$ generates the French word $f$, and $\mathrm{d}$ is the distortion probability that an English word in a certain position will gener-ate a French word in a certain position. This formula is one of many ways in which $p(\mathbf{f}, \mathbf{a} \mid \mathbf{e})$ can be written as the product of a series of conditional probabilities.

In above model, the distortion probability is related with positional preference(word order). Since Korcan is a free order language, the probability is not feasible in English-Korean translation.

Furthermore, the difference between two languages leads to the discordance between words that; the one-to-one correspondence between words gencrally does not keep. The model (1), however, assumed that an English word can be connected with multiple French words, but that cach French word is connected to exactly one English word including; the empty word. In conclusion, many-to-many mappings are not allowed in this model.

According to our experiment, many-to-many mappings exceed $40 \%$ in English and Korean lexical alignments. Only $25.1 \%$ of them can be explained by word for word correspondences. It means that we need a statistical model which can handle phrasal mappings.

In the case of the phrasal mappings, a lot of parameters should be searched even if we restrict the length of word strings. Moreover, in order to properly estimate parameters we need much larger volume of bilingual aligned text than it in word-forword modoling. Even though such a large corpora cxist sometimes, they do not come up with the loxical alignments.

For this problem, we here consider syntactic features which are important in determining structures. A structural feature means here a mapping between tag sequences in bilingual parallel sentences.

If we are concerned with tag sequence alignments, it is possible to estimate statistical parameters in a relatively small size of corpora. As a result, we can remarkably reduce the problem space for possible lexical alignments, a sort of $t$ probability in (1), which improve the complexity of a statistical machine translation model.
If there are similarities between corresponding tag sequences in two language, the structural features would be easily computed or recognized. However, a tag sequence in English can be often translated into a completely different tag sequence in Korcan as follows.

can/MD $\rightarrow \sim e u l /$ ENTR1 su/NNDE1 'iss/AJMA $d a /$ ENTE

It means that similarities of tag features between two languages are not kept all the time and it is necessary to get the most likely tag sequence mappings that reflect structural correspondences between two languages.

In this paper, the tag sequence mappings are obtaind by automatic feature selcction based on the maximum entropy model.

\section{Problem Setting}

In this chapter, we describe how the features are related to the training data. Let $t_{c}$ be an English tag sequence and $t_{k}$ be a Korean tag sequence. Let $\mathcal{T}_{S}$ be the set of all possible tag scquence mappings in a aligned sentence, $S$. We define a feature function (or a feature) as follows:

$$
f\left(t_{e}, t_{k}\right)=\left\{\begin{array}{cc}
1 & \text { pair }\left(t_{c}, t_{k}\right) \in \mathcal{T}_{S} \\
0 & \text { otherwise }
\end{array}\right.
$$

It indicates co-occurrence information between tags appeared in $\mathcal{T}_{S} . f\left(t_{e}, t_{k}\right)$ expresses the information for predicting that $t_{e}$ maps into $t_{k}$. A foature means a sort of information for predicting something. In our model, co-occurrence information on the same aligned sentence is used for a feature, while context is used as a feature in most of systems using maximum entropy. It can be less informative than context. Hence, we considered an initial supervision and feature selection.

Our model starts with initial seed(active) features for mapping extracted by supervision. In the next step, feature pool is constructed from training samples from filtering and only foatures with a large gain to the model are added into active feature set. The final outputs of our model are the set of active fcatures, their gain values, and conditional probabilities of features which maximize the model. The results can be embedded in parameters of statistical machine translation and help to construct structural bilingual text.

Most alignment algorithm consists of two steps: (1) estimate translation probabilities.

(2) use these probabilities to search for most probable alignment path.

Our study is focused on (1), especially the part of tag string alignments.

Next, we will explain the concept of the model. We are concerned with an optimal statistical model which can generate the training samples. Namely, our task is to construct a stochastic model that pro- 
duces output tag sequence $\mathcal{T}_{k}$, given a tag sequence $\mathcal{T}_{c}$. The problem of interest is to use samples of tagged sentences to observe the behavior of the random process. 'The model $p$ estimates the conditional probability that the process will output $t_{e}$, given $t_{k}$. It is chosen out of a set of all allowed probability distributions.

The following steps are employed for our model.

Input: a set $\mathbf{L}$ of POS-labeled bilingual aligned sentences.

1. Make a set $\mathcal{F}$ of correspondence pairs of tag sequences, $\left(t_{e}, t_{k}\right)$ from a small portion of $\mathrm{L}$ by supervision.

2. Set, $\mathcal{F}$ into a set of active features, $\mathcal{A}$.

3. Maximization of parameters, $\lambda$ of active features by IIS(Improved Iterative Scaling) algorithm.

4. Create a feature pool set $\mathcal{P}$ of all possible alignments a $\left(t_{e}, t_{k}\right)$ from takg scquences of samples.

5. Filter $\mathcal{P}$ using frequency and similarity with $\mathcal{A}$.

6. Compute the approximate gains of fatures in $\mathcal{P}$.

7. Select now foatures $(\mathcal{N})$ with a large gain value, and add $\mathcal{A}$.

Output: $p\left(t_{k} \mid t_{c}\right)$ where $\left(t_{c}, t_{k}\right) \in \mathcal{A}$ and their $\lambda_{i}$.

We began with training samples composed of English-Korcan aligned sentence paiss, $(\mathbf{c}, \mathbf{k})$. Since they included long sentences, wo broke them into shorter ones. The length of training sentences was limited to muder 14 on the basis of English. It is reasonable becantse we are interested in not lexical aligmments but tag sequence alignments. 'I'he samples wore tagged using brill's tagger and 'Mouany' that we implemented as a Korean tagger. Figure 1 shows the POS tags we considered. For simplicity, we adjusted some part of Brill's tag sot.

In the supervision step, 700 aligned sentences were used to construct the tag sequences mappings which are reforred to as an active feature set, $\mathcal{A}$. As Figure 2 shows, there are several ways in constructing the correspondences. We chose the third mapping although (1) can be more useful to explain Korcan with predicate-argument structure. Since a subject, of a English sentence is always used for a subject, form in Korean, we exlcuded a subject case from arguments of a predicate. For example, 'they' is only used for a subject form, whereas 'me' is used for a object form and a dative form.

In the next step, training events, $\left(t_{c}, t_{k}\right)$ are constructed to make a feature pool from training samples. The event consists of a tag string $t_{e}$ of a English
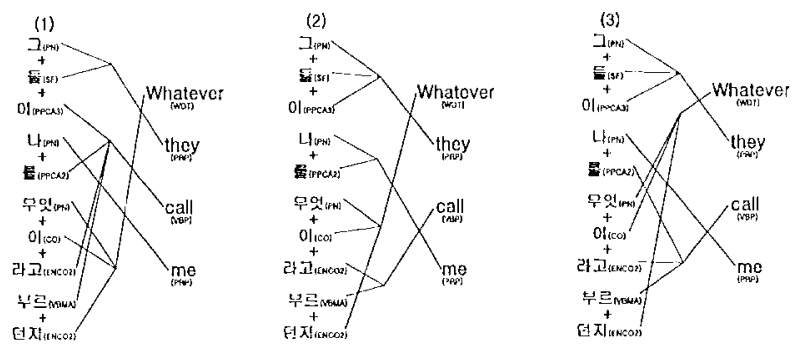

Figure 2: Tag sequence correspondences at the phrase level

P'OS-tagged sentence and a tag string $t_{k}$ of the corresponding Korcan POS-tagged sentence and it can be represented with inclicator functions $f_{i}\left(t_{c}, t_{k}\right)$.

For a given sequence, the features were drawn from all adjacent possible pairs and some interrupted pairs. Only features $\left(t_{e i}, t_{k i}\right)$ out of the foature pool that meet the following conditions are extracted.

- $\#\left(t_{c i}, t_{k i}\right) \geq 3, \#$ is count

- there oxist $t_{k x}$, where $\left(t_{c i}, t_{k x}\right)$ in $\mathcal{A}$ and the similarity (same tang count) of $t_{k i}$ and $t_{k x} \geq 0.6$

Table 1 shows possible features, for a given aligned sentence, 'take her out - gnyeoreul baggeuro deryeogaras.

Since the sct of the structural foatures for alignment modeling is vast, we constructed a maximum cntropy model for $p\left(t_{k} \mid t_{c}\right)$ by the iterative model growing method.

\section{Maximum Entropy}

To explain our mothod, we briefly describe the conc(ut of maximum entropy. Recently, many approaches based on the maximum entropy model have been applied to natural language processing (13crger et al., 1994; Berger et al., 1996; Pietra ot al., 1997).

Suppose a moclel $\mathbf{p}$ which assigns a probability to a random variable. If wo don't have any knowledge, a reasonable solution for $p$ is the most uniform distribution. As some knowledge to estimate the model $p$ are added, the solution space of $p$ are more constrained and the model would be close to the optimal probability model.

For the purpose of getting the optimal probability model, we need to maximize the uniformity under some constraints we have. Here, the constraints are related with features. A feature, $f_{i}$ is usually represented with a binary indicator function. The importance of a foature, $f_{i}$ can be identified by requiring that the model accords with it.

As a constraint, the expected value of $f_{i}$ with respect to the model $p\left(f_{i}\right)$ is supposed to be the same as the expected value of $f_{i}$ with respect to empirical distribution in training samples, $\tilde{p}\left(f_{i}\right)$. 


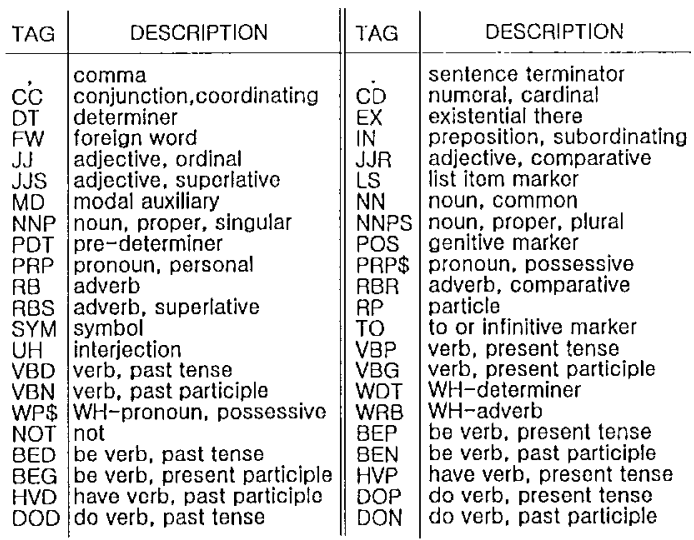

\begin{tabular}{|c|c|c|c|}
\hline TAG & POS & TAG & POS \\
\hline $\begin{array}{l}\text { NNIN1 } \\
\text { NNIN2 } \\
\text { NNDE1 } \\
\text { NNDE2 } \\
\text { PN } \\
\text { NU } \\
\text { VBMA } \\
\text { AJMA } \\
\text { CO } \\
\text { AX } \\
\text { ADCO } \\
\text { ADSE } \\
\text { CJ } \\
\text { ANCO } \\
\text { ANDE } \\
\text { ANNU } \\
\text { EX } \\
\text { LQ } \\
\text { RQ } \\
\text { SY }\end{array}$ & $\begin{array}{l}\text { proper noun } \\
\text { common noun } \\
\text { common-dependent noun } \\
\text { unit-dependent noun } \\
\text { pronoun } \\
\text { number } \\
\text { verb } \\
\text { adjective } \\
\text { copula } \\
\text { auxiliary verb } \\
\text { constituent adverb } \\
\text { sentential adverb } \\
\text { conjunctive adverb } \\
\text { configurative adnominal } \\
\text { demonstrative adnominal } \\
\text { numeral adnominal } \\
\text { exclamination } \\
\text { left quotation mark } \\
\text { right quotation mark } \\
\text { symbols }\end{array}$ & $\begin{array}{l}\text { PPCA1 } \\
\text { PPCA2 } \\
\text { PPCA3 } \\
\text { PPCA4 } \\
\text { PPAD } \\
\text { PPCI } \\
\text { PPAU } \\
\text { ENTE } \\
\text { ENCO1 } \\
\text { ENCO2 } \\
\text { ENCO3 } \\
\text { ENTR1 } \\
\text { ENTR2 } \\
\text { ENTR3 } \\
\text { ENCM } \\
\text { PE } \\
\text { SF } \\
\text { PF } \\
\text { CM } \\
\text { SC }\end{array}$ & $\begin{array}{l}\text { nominative postposition } \\
\text { accusative postposition } \\
\text { possessive postposition } \\
\text { vocative postposition } \\
\text { adverbial postposition } \\
\text { conjunctive postposition } \\
\text { auxiliary postposition } \\
\text { final ending } \\
\text { coordinate ending } \\
\text { subordinate ending } \\
\text { auxiliary ending } \\
\text { adnominal ending } \\
\text { nominal ending } \\
\text { adverbial ending } \\
\text { ending+postposition } \\
\text { pre-ending } \\
\text { suffix } \\
\text { prefix } \\
\text { comma } \\
\text { termination }\end{array}$ \\
\hline
\end{tabular}

Figure 1: English Tags (left) and Korean Tags (right)

\begin{tabular}{|c|c|}
\hline English Jag Sequences & Korean Tag Sequences \\
\hline 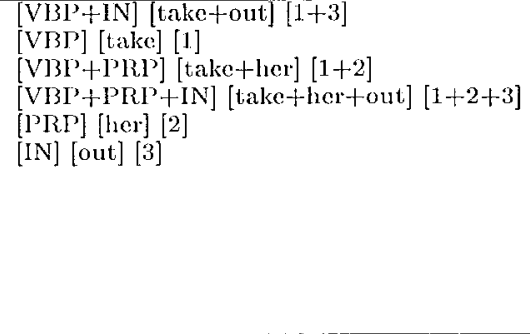 & 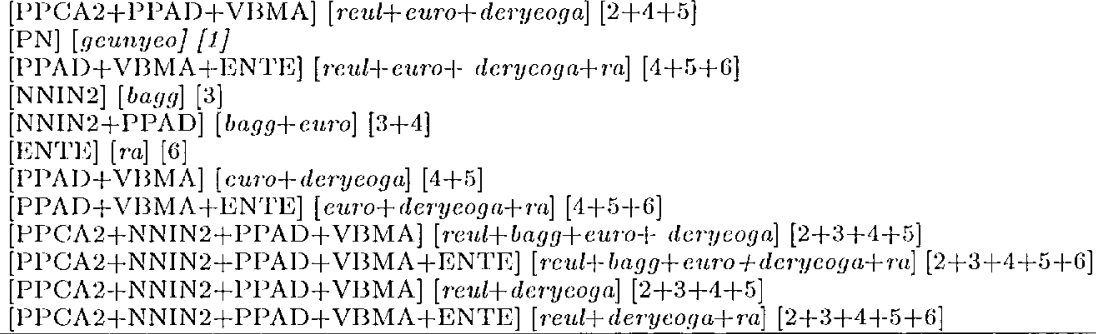 \\
\hline
\end{tabular}

Table 1: possible tag sequences

In sum, the maximum entropy framework finds the model which has highest entropy (most uniform), given constraints. It is related to the constraincel optimization. To select a model from a constrained set $C$ of allowed probability distributions, the model $p_{\star} \in C$ with maximum entropy $H(p)$ is chosen.

In general, for the constrained optimization prob-lcm, Lagrange multipliers of the number of features can be used. However, it was proved that the model with maximum entropy is equivalent to the model that maximizes the log likclihood of the training; samples like (2) if wo can assume it as an exponential model.

In (2), the left side is Lagrangian of the conditional entropy and the right side is maximum loglikelihood. We use the right side equation of (2) to select $\lambda_{\star}$ for the best model $p_{\star}$.

$$
\begin{array}{r}
\operatorname{argmax}_{\lambda_{i}}\left(-\sum_{x, y} \tilde{p}(x) p(y \mid x) \log p(y \mid x)+\lambda_{i}\left(p\left(f_{i}\right)-\tilde{p}\left(f_{i}\right)\right)\right) \\
=\operatorname{argmax}_{\lambda_{i}} \sum_{x, y} \tilde{p}(x, y) \log p(y \mid x)
\end{array}
$$

Since $\lambda_{\star}$ cannot be found analytically, we use the following improved itcrative scaling algorithm to compute $\lambda_{\star}$ of $n$ active features in $\mathcal{A}$ in total samples.

1. Start with $\lambda_{i}=0$ for all $i \in\{1,2, \ldots, n\}$
2. Do for cach $i \in\{1,2, \ldots, n\}$ :

(a) Let $\Delta \lambda_{i}$ be the solution to the log likelihood

(b) Update the value of $\lambda_{i}$ into $\lambda_{i}+\Delta \lambda_{i}$,

$$
\begin{aligned}
& \text { where } \Delta \lambda_{i}=\log \frac{\sum_{x, y} \bar{p}(x, y) f_{i}(x, y)}{\sum_{x, y} \bar{p}(x) p_{\lambda}(y \mid x) f_{i}(x, y)} \\
& p_{\lambda}(y \mid x)=\frac{1}{Z_{\lambda}(x)} e^{\left(\sum_{i} \lambda_{i} f_{i}(x, y)\right)}, \\
& Z_{\lambda}(x)=\sum_{y} e^{\left(\sum_{i} \lambda_{i} f_{i}(x, y)\right)}
\end{aligned}
$$

3. Stop if not all the $\lambda_{i}$ have converged, otherwise go to stcp 2

The exponential model is represented as (3). Herc, $\lambda_{i}$ is the weight of feature $f_{i}$. In our model, since only one feature is applicd to each pair of $x$ and $y$, it can be represented as (4) and $f_{i}$ is the feature related with $\mathrm{x}$ and $\mathrm{y}$.

$$
\begin{gathered}
\tilde{p}(y \mid x)=\frac{\sum_{i} e^{\lambda_{i} f_{i}(x, y)}}{\sum_{y} e^{\sum_{i} \lambda_{i} f_{i}(x, y)}} \\
\tilde{p}(y \mid x)=\frac{e^{\lambda_{i} f_{i}(x, y)}}{\sum_{y} e^{\lambda_{i} f_{i}(x, y)}}
\end{gathered}
$$




\section{$5 \quad$ Feature selection}

Only a small subset of features will be employed in a model by selecting useful foatures from the feature pool $P$. Let $p_{\mathcal{A}}$ be the optimal model constrained by a set of active features $\mathcal{A}$ and $\mathcal{A} \cup f_{i}$ be $\mathcal{A} f_{i}$. Let $p_{\mathcal{A} f_{i}}$ be the optimal model in the space of probability distribution $C\left(\mathcal{A} f_{i}\right)$. The optinal model can be represented as (5). Here, the optimal model means a maximum entropy model.

$$
\begin{array}{r}
p_{\mathcal{A} f_{i}}^{\alpha}=\frac{1}{Z_{\alpha}(x)} p_{\mathcal{A}}(y \mid x) e^{\alpha f_{i}(x, y)} \\
Z_{\alpha}(x)=\sum_{y} p_{\mathcal{A}}(y \mid x) e^{\alpha \gamma f_{i}(x, y)}
\end{array}
$$

The inprovement of the model regarding the addition of a single foature $f_{i}$ can be estimated by moasuring the difference of maximum log-likelihood between $L\left(p_{\mathcal{A} f_{i}}\right)$ and $L\left(p_{\mathcal{A}}\right)$. We denote the gain of feature $f_{i}$ by $\Delta\left(\mathcal{A} f_{i}\right)$ and it can be represented in (6).

$$
\begin{aligned}
\Delta\left(\mathcal{A} f_{i}\right) \equiv & \max _{\alpha} G_{\mathcal{A} f_{i}}(\alpha) \\
G_{\mathcal{A} f_{i}}(\alpha) \equiv & I_{i}\left(p_{\mathcal{A}} f_{i}\right)-I_{(}\left(p_{\mathcal{A}}\right) \\
= & -\sum_{x} \tilde{p}(x) \sum_{y} p_{\mathcal{A}}(y \mid x) e^{\alpha f_{i}(x, y)} \\
& +\alpha \tilde{p}\left(f_{i}\right)
\end{aligned}
$$

Note that a model $p_{\mathcal{A}}$ has a set of paramoters $\lambda$ which means woights of features. The model $p_{\mathcal{A}} f_{i}$ contains the parameters and the now parameter $a$ with respect to the feature $f_{i}$. Whon adding a now foature to $A$, the optimal values of all parameters of probability distribution change. To make the computation of foature selection tractable, we appuseximate that the addition of a feature $f_{i}$ aflects only the single parameter $\alpha$, as shown in (5).

The following algorithm is used for computing the gain of the model with respect to $f_{i}$. We referred to the studies of (Berger et al., 1996; Pictra et al. 1997). We skip the detailed contents and proofs.

1. Let

$$
r=\left\{\begin{array}{l}
1 . \\
-1 \quad \text { if } \tilde{p}\left(f_{i}\right) \leq p_{\mathcal{A}}\left(f_{i}\right)
\end{array}\right.
$$

2. Set $\alpha_{0}=0$

3. Repeat the following until $G_{\mathcal{A} f_{i}}\left(\alpha_{n}\right)$ has converged :

Compute $\alpha_{n+1}$ from $\alpha_{n}$ using

$$
\begin{aligned}
& \alpha_{n+1}=\alpha_{n}+\frac{1}{r} \log \left(1-\frac{1}{r} \frac{G_{\mathcal{A} f_{i}}^{\prime}\left(\alpha_{n}\right)}{G_{\mathcal{A} f_{i}}^{\prime \prime}\left(\alpha_{n}\right)}\right) \\
& \text { Compute } G_{\mathcal{A} f_{i}}\left(\alpha_{n+1}\right) \text { using } \\
& G_{\mathcal{A} f_{i}}(\alpha)=-\sum_{x} \tilde{p}(x) \log Z_{\alpha}(x)+\alpha \tilde{p}\left(f_{i}\right), \\
& G_{\mathcal{A} f_{i}}^{\prime}(\alpha)=\tilde{p}\left(f_{i}\right)-\sum_{x} \tilde{p}(x) M(x) \\
& G_{\mathcal{A} f_{i}}^{\prime \prime}(\alpha)=-\sum_{x} \tilde{p}(x) p_{\mathcal{A} f_{i}}^{\alpha}\left(\left(f_{i}-M(x)\right)^{2} \mid x\right)
\end{aligned}
$$

\begin{tabular}{c|l|c|c}
\hline set & description & $\begin{array}{c}\text { \#f of disjoint } \\
\text { features }\end{array}$ & $\begin{array}{c}\text { total } \\
\text { events }\end{array}$ \\
\hline $\mathrm{A}$ & active features & 1483 & 4113 \\
$\mathrm{P}$ & fouture candidates & 3172 & 63773 \\
$\mathrm{~N}$ & new foalures & 97 & 5503 \\
\hline
\end{tabular}

Table 2: Summery of Features Sclected

$$
\begin{aligned}
& \text { where } \alpha=\alpha_{n+1} \text {, } \\
& \mathcal{A} f_{i}=\mathcal{A} \cup f_{i} \\
& M(x) \equiv p_{\mathcal{A} f_{i}}^{\alpha}\left(f_{i} \mid x\right), \\
& p_{\mathcal{A} f_{i}}^{n}\left(f_{i} \mid x\right) \doteq \sum_{y} p_{\mathcal{A} f_{i}}^{\alpha}(y \mid x) f_{i}(x, y) \\
& \text { 4. Set } \sim \Delta L\left(\mathcal{A} f_{i}\right) \leftarrow G_{\mathcal{A} f_{i}}\left(\alpha_{n}\right)
\end{aligned}
$$

This algorithm is itcratively computed using Netwon's method. We can recognize the importance of a feature with the gain value. As mentioned above, it uncans how much the feature accords with the model. We viewed the feature as the information that $t_{k}$ and $t_{\text {e occur togethor. }}$

\section{$6 \quad$ Experimental results}

The total samples consists of 3,000 aligned sentence pairs of English-Korcan, which were extracted from nows on the web site of 'Korea Times' and a nnagezino for Lnglish learning.

In the initial step, we manually constucted the correspondences of tag sequences with 700 POStagged scntence pairs. In the supervision step, we extracted 1,483 correct tag sequence correspondonces as shown in Table 2 , and it work as activo features. As a feature pool, 3,172 disjoint features of tiag sequence mappings were retrieved. It is very important to make atomic features.

We maximized $\lambda$ of active foatures with respect to total samples using improved the iterative scaling algorithm. Figure 3 shows $\lambda_{i}$ of cach foature $f\left(t_{B E P}+J J, t_{k}\right) \in \mathcal{A}$. 'There are many correspondence patterns with respect to the Englsh tag string, 'BEP+JJ'.

Note that $p\left(t_{k} \mid t_{e}\right)$ is computed by the exponential model of (4) and the conditional probability is the same with empirical probability in (7). Since the value of $p(y \mid x)$ shows the maximum likelihood, it is proved that cach $\lambda$ was converged correctly.

$$
p(y \mid x) \equiv \frac{\# \text { of }(x, y) \text { occurs in sample }}{\text { number of times of } x}
$$

In feature selection step, we chose useful features with the gain threshold of 0.008 . Figure 4 shows some feaures with a large gain. Among them, tag sequences mapping including ' $R B$ ' are erroncous. It means that position of adverb in Korean is very complicated to handle. Also, proper nown in English aligned common nouns in Korean 


\begin{tabular}{|c|c|c|c|c|c|c|}
\hline \multicolumn{2}{|r|}{ Feature $(x, y)$} & \multirow[t]{2}{*}{1} & \multirow[t]{2}{*}{$c(x, y)$} & \multirow[t]{2}{*}{$p(y \mid x)$} & \multicolumn{2}{|c|}{ Example } \\
\hline English & Korean & & & & English & Korean \\
\hline $\begin{array}{l}B E P+J J \\
B E P+J J \\
B E P+J J \\
B E P+J J \\
B E P+J J \\
B E P+J J \\
B E P+J J \\
B E P+J J \\
B E P+J J \\
B E P+J J \\
B E P+J J\end{array}$ & $\begin{array}{l}\text { VBMA+ENCO3+AX+ENTE } \\
\text { VBMA } \\
\text { AJMA } \\
\text { AJMA+ENTE } \\
\text { VBMA+ENTE } \\
\text { NNIN2+CO } \\
\text { NNIN2+CO+VBMA } \\
\text { NNIN2+PPCA1+VBMA+ENTE } \\
\text { NNIN2+CO+ENTE } \\
\text { NNIN2+PPCA2+AX+ENTE } \\
\text { NNIN2+PPCA1+VBMA }\end{array}$ & $\begin{array}{l}10.1369 \\
8.8520 \\
8.6787 \\
8.2628 \\
7.2379 \\
7.1372 \\
6.9909 \\
6.8402 \\
6.8308 \\
6.4256 \\
6.4250\end{array}$ & $\begin{array}{l}162.00 \\
45.00 \\
39.00 \\
25.00 \\
9.00 \\
8.00 \\
7.00 \\
6.00 \\
6.00 \\
4.00 \\
4.00\end{array}$ & $\begin{array}{l}0.4247 \\
0.1180 \\
0.0996 \\
0.0655 \\
0.0236 \\
0.0210 \\
0.0183 \\
0.0157 \\
0.0157 \\
0.0105 \\
0.0105\end{array}$ & $\begin{array}{l}\text { are+prepared } \\
\text { are+careful } \\
\text { am+healthy } \\
\text { is+new } \\
\text { am+sure } \\
\text { am+rich } \\
\text { istselfish } \\
\text { istpatriotic } \\
\text { istreasonable } \\
\text { istreprehensible } \\
\text { isthelpful }\end{array}$ & 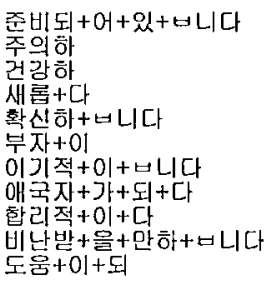 \\
\hline
\end{tabular}

Figure 3: $\lambda$ of active features in $\mathcal{A}$

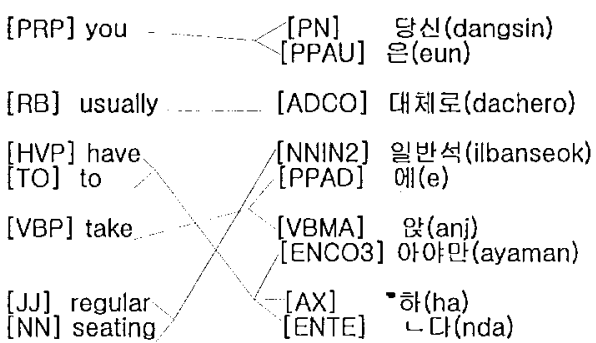

Figure 5: Best Lexical alignment

because of tagging errors. Note that in the case of 'PN+PPCA2+PPAD+VBMA', it is not an adjacent string but an interrupted string. It means that a verb in English generally map to a verb taking as argument the accusative and adverbial postposition in Korean.

One way of testing usefulness of our method is to construct structured aligned bilingual sentences. Table 3 shows lexical alignments using tag sequence alignments drawn from our algorithm for a given sentence, 'you usually have to take regular seating - dangsineun dachero illanseoke anjayaman handa' and Figure 5 shows the best lexical alignment of the sentence.

We conducted the experiment on 100 sentences composed of words in length 14 or less and simply chose the most likely paths. As the result, the accuray was about $71.1 \%$. It shows that we can partly use the tag sequence alignments for lexical alignments. We will extend the structural mapping model with consideration to the lexical information. The parameters, the conditional probabilities about stuctural mappings will be embedded in a statistical model. Table 4 shows conditional probabilities of some features according to 'DT+NN'. In general, determiner is translated into NULL or adnominal word in Korean.

\section{Conclusion}

When aligning English-Korean sentences, the differences of word order and word unit require structural information. For this reason, we tried structural tag

\begin{tabular}{c|c|c}
$t_{\mathrm{e}}$ & $\iota_{k}$ & $p\left(t_{k} \mid t_{e}\right)$ \\
\hline D'I+NN & NNIN2 & 0.524131 \\
DT+NN & ANISI+NNIN2 & 0.15161 \\
DT+NN & ANNU+NNDE2 & 0.091036 \\
D'T+NN & NNIN2+PPCA1 & 0.06351 .5 \\
DT+NN & NNIN2+NNIN2 & 0.058322 \\
DT+NN & NNIN2+PPAU & 0.05708 \\
D'T+NN & ADCO & 0.049622 \\
etc & etc &
\end{tabular}

Table 4: Conditional Probability

string mapping using maximum entropy modeling and feature selection concept. We devised a model that genorates a English tag string given a Korean tag string. From initial active structural features, uscful features are extended by feature selection. The retrieved features and parameters can be embedded in statistical machine translation and reduce the complexity of searching. We showed that they can helpful to construct structured aligned bilingual sentences.

\section{References}

Adam L. Berger, Peter F. Brown, Stephen A. Della Pietra, Vincent, J. Della Pictra, John R. Gillett, John D. Lafferty, Robert L. Mercer, Harry Printz, and Lubos Ures. 1994. The Candie system for machine translation. In Procecdings of the ARPA Conference on Human Language Technology, Plainsborough, New Jersey.

Adam L. Berger, Stephen A. Della Pietra, and Vincent J. Della Pietra. 1996. A maximum entropy approach to natural language processing. Computational Linguistics, 22(1):39-73.

Peter F. Brown, John Cocke, Stephen A. Della Pietra, Vincent J. Della Pietra, Fredrick Jelinek, John D. Lafferty, Robert L. Mercer, and Paul S. Roossin. 1990. A statistical approach to machine translation. Computational Linguistics, 16(2):7985

Peter F. Brown, Stephen A. Della Pietra, Vincent J. Della Pietra, Robert L. Mercer. 1993. The mathematics of statistical machine translation: pa- 


\begin{tabular}{|c|c|c|c|c|c|c|}
\hline \multicolumn{2}{|r|}{ Feature $(x, y)$} & \multirow[t]{2}{*}{$x$} & \multirow{2}{*}{$P(y \mid x)$} & $\triangle L\left(A f_{1}\right)$ & \multicolumn{2}{|c|}{ Example } \\
\hline$x$ & $Y$ & & & & English & $\mathrm{Ko}$ \\
\hline $\mathrm{BP}+\mathrm{PRP}+\mathrm{TO}$ & $\mathrm{PN}+\mathrm{PPCA} 2+\mathrm{PPA}+\mathrm{VBMA}$ & 9.8687 & $0 . \overline{17} \overline{22}$ & 0.0194 & $\operatorname{sen}$ & 가를+ \\
\hline$E P+R B R+I N$ & JMA & 9.6780 & 0.3 & 0.0192 & is $+m$ & $\sim$ 보다 + 믄 \\
\hline $\begin{array}{l}P T+G D \\
J+\mathbb{N}\end{array}$ & $E 2$ & 9 & & 0.0190 & thet & 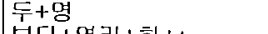 \\
\hline TO & AA+ENTR1 & & & 0. & $\mathrm{sm}$ & 영: \\
\hline ro & $\mathrm{PP}$ & & & & $\begin{array}{l}\text { se } \\
\text { is }\end{array}$ & $+2 \sim$ 늘 + 나드 \\
\hline $\mathrm{EH}, 2 \mathrm{C}$ & $\mathrm{PP}$ & & & & are & $\tilde{0}$ \\
\hline $\mathrm{vr}$ & & & & & $|B|$ & \\
\hline NF & IIN2 & & & & Hal & 드+대학 \\
\hline$R P$ & & & & & to & 7 \\
\hline RP & PN & & & & to + & 그t \\
\hline & & & & & & \\
\hline & . & & & & should & 하 \\
\hline & & & & & Englis & 영응 \\
\hline & $P P$ & 8. & & 0.0 & request+to+send & 을+보내 +라 \\
\hline$+\quad+\mathrm{VBI}$ & $M A+P E+E S$ & 11 & & $\begin{array}{l}73 \\
73\end{array}$ & $\begin{array}{l}\text { wast throwntto } \\
\text { were+sent+to }\end{array}$ & ～예게+턴졎 \\
\hline & & & & & & \\
\hline
\end{tabular}

Figure 4: Some features with a large gain

\begin{tabular}{|c|c|c|}
\hline 'ladg alignment: & Conditional & Lexical alignment \\
\hline PRI : PN+PI'AU & 0.150109 & you : dangsint-cun \\
\hline $13.13: \Lambda I) C()$ & 0.1421 .93 & ustaally : dachero \\
\hline $\mathrm{RI} 3: \mathrm{NNIN} 2+\mathrm{I}^{\prime} \mathrm{l}(\mathrm{AI})$ & 0.038105 & usually : ilbanseokte \\
\hline IIVI $+\mathrm{T}^{\prime} \mathrm{O}: \mathrm{ENCO} 3+-\Lambda \mathrm{X}+\mathrm{WN} \mathrm{N}^{\prime}$ & 0.982839 & have+to : ayamanthanda \\
\hline$V B 1^{\prime}: I^{\prime} P^{2} A D+V B M A$ & $0.050 \overline{224}$ & take: $\mathrm{c}+\mathrm{anj}$ \\
\hline 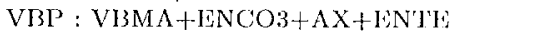 & 0.011110 & take: anjay+aman+ha+nda \\
\hline 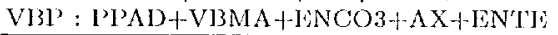 & 0.001851 & take: et-anjayamanthancla \\
\hline$\left.\left.V B 1^{\prime}+J I J: N N I N 2+I^{\prime} P A\right]\right)-V I 3 M A$ & 0.057657 & take-tregular : ilbanseok +e+anj \\
\hline$J J+N N:$ NNIN2 & $0.5 \overline{81791}$ & regular+seating : ilbanseok \\
\hline
\end{tabular}

Table 3: Iexical aligmments using tag alignments

rancter estimation. Computational Linguistics, 19(2):263-311.

Stanley F. Chen. 1993. Aligning sentences in bilingual corpora using lexical information. In Proceedings of ACL 31, 9-16.

A. P. Dompster, N. M. Laird and D. B. Rubin. 1976. Maximm likelihood from incomplete clata via the EM algorithm. The Royal Statistics Society, 39(B) 205-237.

William A. Gale, Konneth W. Church. 1993. A program for aligning sentences in bilingual corpora. Computational Linguistics, 19:75-102.

Trederick Jelinek. 1997. Statistical Methods for Sycech Recognition MIT Press.

Marin Kay, Martin Roscheisen. 1993. Texttranslation aligmmont. Computational Linguistics, 19:121-142.

Julian Kupiec. 1993. An algorithm for finding noun phrase correspondences in bilingual corpora. In Procecdings of ACL 31, 17-22.

Yuji Matsumoto, Hiroyuki Ishimoto, Takchito Utsuro. 1993. Structural matching of parallel texts. In Proceedings of $A C L$ 31, 23-30.

I. Dan Molamed. 1997. A word-to-word model of translation equivalence. In Proceedings of $A C L$ 35/EACL 8, 16-23.

Franz Josef Och and Hans Wobcr. 1998. Improving Statistical Natural Language Translation with Categories and Rules. In Procecdings of $A C L$ 36/COLING, 985-989.
Steplicn A. Della Pietra, Vincent; J. Della Pietra, John D. Laflerty. 1997. Inducing features of random ficlds. IELE Transactions on Pattern Analysis and Machine Intelligence, 19(4):380-393.

Frank Smadja, Kathlecn R. McKcown, and Vasileios Hatzivassiloglou. 1996. Translating collocations for bilingual loxicons: A statistical approach. Computational Linguistics, 22(1):1-38.

Kongo Sato 1998. Maximum Entropy Modd Leaning of the Thanslation Rules. In Proccedings of ACL 36/COLING, 1171-1175.

Jung H. Shin, Young S. Han, and Key-Sun Choi. 1996. Bilingual knowledge acquisition from Korcan-English parallel corpus using alignment method. In Proceedings of COLING 96.

C. Tillmann, S. Vogel, H. Ney, and A. Zubiaga. 1997. A DP based scarch using monotone alignments in statistical translation. In Proceedings of ACL 35/EACL 8, 289-296.

Ye-Yi Wang and Alox Waibel. 1997. Decoding algorithm in statistical machine translation. In Proccedings of $A C L$ 35/EACL 8, 366-372.

Ye-Yi Wang and Alex Waibel. 1998. Modeling with structures in machine translation. In Procecdings of ACL $36 / C O L I N G$

Dekai Wu 1996. A polynomial-time algorithm for statistical machine translation. In Proceeding of ACL 34. 